\title{
Correction to: DNMT3b/OCT4 expression confers sorafenib resistance and poor prognosis of hepatocellular carcinoma through IL-6/STAT3 regulation
}

Ssu-Chuan Lai ${ }^{1,2}$, Yu-Ting Su ${ }^{1,2,3+}$, Ching-Chi Chi ${ }^{4,5+}$, Yung-Che Kuo ${ }^{1,2,3}$, Kam-Fai Lee ${ }^{6}$, Yu-Chih Wu ${ }^{3,7}$, Pei-Chi Lan ${ }^{3}$, Muh-Hwa Yang ${ }^{8,9}$, Te-Sheng Chang ${ }^{10,11^{*}}$ and Yen-Hua Huang ${ }^{1,2,3,12,13,14,15^{*}}$

\section{Correction to: J Exp Clin Cancer Res (2019) 38:474 https://doi.org/10.1186/s13046-019-1442-2}

In the original publication of this article [1], labelling within Fig. 7a was incorrect. The updated figure is shown below, with 'DMT1' now corrected to read 'DNMT1'.

The authors sincerely apologize for the inconvenience caused to the readers.

\footnotetext{
Author details

'Graduate Institute of Medical Sciences, College of Medicine, Taipei Medical University, Taipei 11031, Taiwan. ${ }^{2}$ Department of Biochemistry and Molecular Cell Biology, School of Medicine, College of Medicine, Taipei Medical University, Taipei 11031, Taiwan. ${ }^{3}$ TMU Research Center for Cell Therapy and Regeneration Medicine, Taipei Medical University, Taipei 11031, Taiwan. ${ }^{4}$ Department of Dermatology, Chang Gung Memorial Hospital, Linkou, Taoyuan 33305, Taiwan. ${ }^{5}$ College of Medicine, Chang Gung University, Taoyuan 33302, Taiwan. ${ }^{6}$ Department of Pathology, Chang Gung Memorial Hospital, Chiayi 61363, Taiwan. ${ }^{7}$ School of Respiratory Therapy, College of Medicine, Taipei Medical University, Taipei 11031, Taiwan. ${ }^{8}$ Institute of Clinical Medicine, College of Medicine, National Yang Ming University, Taipei 11221, Taiwan. ${ }^{9}$ Division of Medical Oncology, Taipei Veterans General Hospital, Taipei 11217, Taiwan. ${ }^{10}$ School of Traditional Chinese Medicine, College of Medicine, Chang Gung University, Taoyuan 33382, Taiwan. ${ }^{11}$ Division of Internal Medicine, Department of Gastroenterology and Hepatology, Chang Gung Memorial Hospital, Chiayi 61363, Taiwan. ${ }^{12}$ International PhD Program for Cell Therapy and Regeneration Medicine, College of Medicine, Taipei Medical University, Taipei 11031, Taiwan. ${ }^{13}$ Center for Reproductive Medicine, Taipei Medical University Hospital, Taipei Medical University, Taipei 11031, Taiwan. ${ }^{14} \mathrm{Ph}$.D. Program for Translational Medicine, College of Medical Science and Technology, Taipei Medical University, Taipei 11031, Taiwan. ${ }^{15}$ Comprehensive Cancer Center of Taipei Medical University, Taipei 11031, Taiwan.
}

The original article can be found online at https://doi.org/10.1186/s13046019-1442-2

*Correspondence: cgmh3621@cgmh.org.tw; rita1204@tmu.edu.tw

${ }^{\dagger} \mathrm{Yu}$-Ting Su and Ching-Chi Chi contributed equally to this work.

${ }^{10}$ School of Traditional Chinese Medicine, College of Medicine, Chang Gung University, Taoyuan 33382, Taiwan

${ }^{1}$ Graduate Institute of Medical Sciences, College of Medicine, Taipei Medical University, Taipei 11031, Taiwan

Full list of author information is available at the end of the article
Published online: 13 January 2020

\section{Reference}

1. Lai, et al. DNMT3b/OCT4 expression confers sorafenib resistance and poor prognosis of hepatocellular carcinoma through IL-6/STAT3 regulation. J Exp Clin Cancer Res. 2019;38:474. https://doi.org/10.1186/s13046-019-1442-2.

(c) The Author(s). 2020 Open Access This article is distributed under the terms of the Creative Commons Attribution 4.0 International License (http://creativecommons.org/licenses/by/4.0/), which permits unrestricted use, distribution, and reproduction in any medium, provided you give appropriate credit to the original author(s) and the source, provide a link to the Creative Commons license, and indicate if changes were made. The Creative Commons Public Domain Dedication waiver (http://creativecommons.org/publicdomain/zero/1.0/) applies to the data made available in this article, unless otherwise stated. 
a

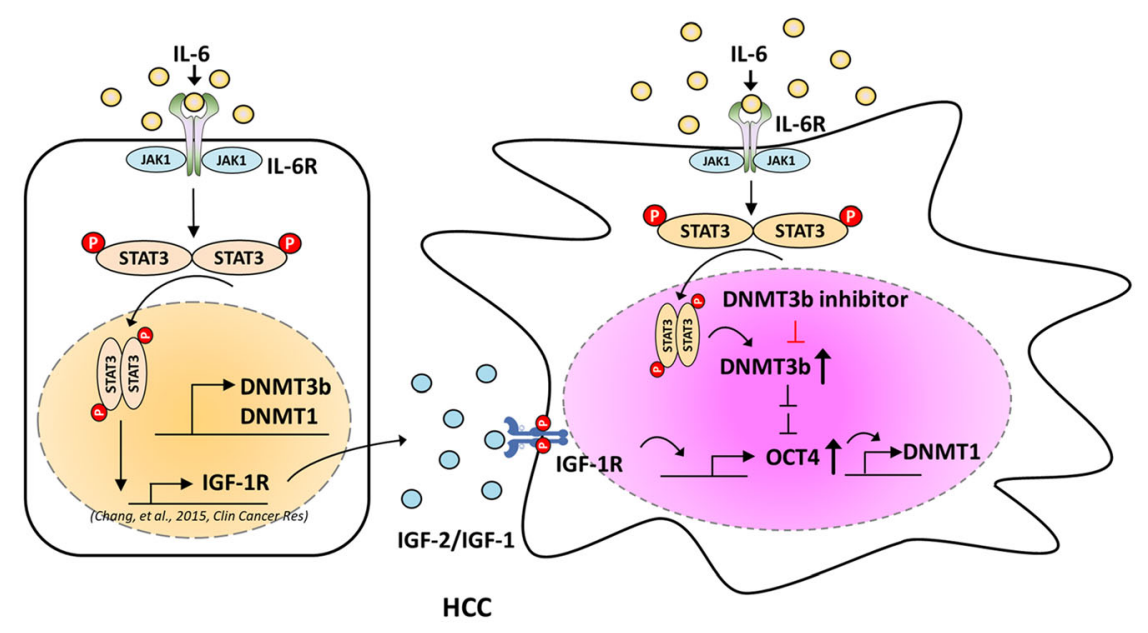

b

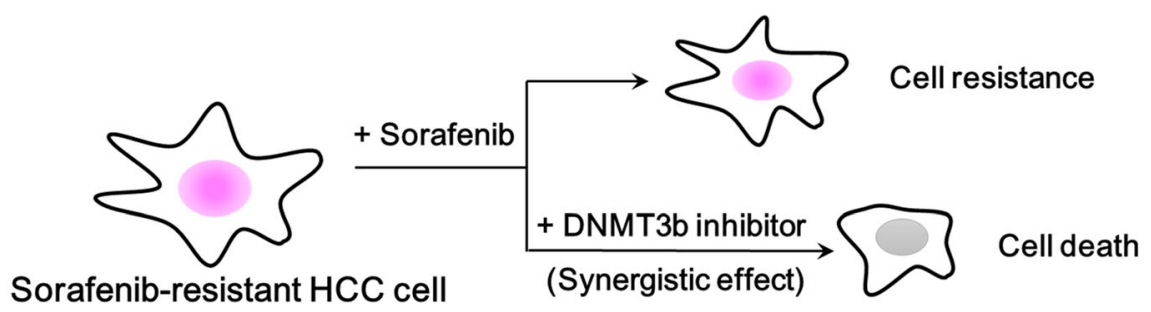

Fig. 7 IL-6 increases the expression of OCT4 through DNMT3b and IGF-1R activation in human HCC. a Model of how IL-6 increases theexpression of OCT4 through p-STAT3-DNMT3b-OCT4-DNMT1 activation in human HCC. $\mathbf{b}$ The combination use of nanaomycin and sorafenibsynergistically suppresses the cell proliferation of sorafenib resistant HCC cells 